PROCEEDINGS OF THE

AMERICAN MATHEMATICAL SOCIETY

Volume 125, Number 5, May 1997, Pages 1363-1366

S 0002-9939(97)03858-6

\title{
APPROXIMATION OF FIXED POINTS OF A STRICTLY PSEUDOCONTRACTIVE MAPPING
}

\author{
LIWEI LIU
}

(Communicated by Palle E. T. Jorgensen)

\begin{abstract}
A fixed point of the strictly pseudocontractive mapping is obtained as the limit of an iteratively constructed sequence with error estimation in general Banach spaces.
\end{abstract}

Let $X$ be a Banach space. A mapping $T$ is said to be strictly pseudocontractive if there exists a number $t>1$ such that the inequality

$$
\|x-y\| \leq\|(1+r)(x-y)-r t(T x-T y)\|
$$

holds for all $x, y \in D(T)$ and $r>0$. We denote by $J$ the normalized duality mapping from $X$ to $2^{X^{*}}$ given by

$$
J x=\left\{f^{*} \in X^{*}:\left\|f^{*}\right\|^{2}=\|x\|^{2}=\operatorname{Re}\left\langle x, f^{*}\right\rangle\right\} .
$$

A mapping $T$ is said to be strongly accretive (see, e.g., $[1,2]$ ) if there exists a positive number $k$ such that for each $x, y \in D(T)$ there is $j \in J(x-y)$ such that

$$
\langle T x-T y, j\rangle \geq k\|x-y\|^{2} .
$$

The inequality (1) implies the inequality

$$
\|x-y\| \leq\|x-y+r[(T-k I) x-(T-k I) y]\| .
$$

Without loss of generality, we can assume $k \in(0,1)$.

Lemma ([6]). Let $X$ be a Banach space, $K$ a subset of $X$, and $T: K \rightarrow K$. Then $T$ is a strictly pseudocontractive mapping if and only if $I-T$ is a strongly accretive mapping, i.e., the inequality

$$
\|x-y\| \leq\|x-y+r[(I-T-k I) x-(I-T-k I) y]\|
$$

holds for any $x, y \in K$ and $r>0$, where $k=(t-1) / t$.

In this paper, we prove by the inequality (2) that the Mann iteration process converges strongly to the unique fixed point of a Lipschitzian and strictly pseudocontractive mapping. Our results extend corresponding results of $[1,2,3,4,5]$ to the general Banach spaces. Furthermore, error estimates are also given.

The set of fixed points of the mapping $T$ is denoted by $F(T)$. The Lipschitzian constant of $T$ is denoted by $L(\geq 1)$.

Received by the editors July 11, 1995 and, in revised form, November 3, 1995.

1991 Mathematics Subject Classification. Primary 47H17.

Key words and phrases. Strictly pseudocontractive mapping, iterative process. 
Theorem 1. Let $X$ be a Banach space, and let $K$ be a nonempty closed convex and bounded subset of $X$. Let $T: K \rightarrow K$ be a Lipschitzian strictly pseudocontractive mapping. If $F(T) \neq \varnothing$, then $\left\{x_{n}\right\} \subset K$ generated by $x_{1} \in K$,

$$
x_{n+1}=\left(1-\alpha_{n}\right) x_{n}+a_{n} T x_{n}
$$

with $\left\{\alpha_{n}\right\} \subset(0,1]$ satisfying

$$
\sum_{n=1}^{\infty} a_{n}=\infty, \quad \alpha_{n} \rightarrow 0,
$$

strongly converges to $q \in F(T)$ and $F(T)$ is a single set.

Proof. Let $q$ be a fixed point of $T$. Since $T$ is a strictly pseudocontractive mapping, then $I-T$ is strongly accretive, i.e., the inequality (2) holds for any $x, y \in K$ and $r>0$.

From the definition of $\left\{x_{n}\right\}$, we have

$$
\begin{aligned}
x_{n}= & x_{n+1}+\alpha_{n} x_{n}-\alpha_{n} T x_{n} \\
= & \left(1+\alpha_{n}\right) x_{n+1}+\alpha_{n}(I-T-k I) x_{n+1}-(2-k) \alpha_{n} x_{n+1} \\
\quad & \quad+\alpha_{n} x_{n}+\alpha_{n}\left(T x_{n+1}-T x_{n}\right) \\
= & \left(1+\alpha_{n}\right) x_{n+1}+\alpha_{n}(I-T-k I) x_{n+1}-(2-k) \alpha_{n}\left[\left(1-\alpha_{n}\right) x_{n}+\alpha_{n} T x_{n}\right] \\
\quad & \quad+\alpha_{n} x_{n}+\alpha_{n}\left(T x_{n+1}-T x_{n}\right) \\
= & \left(1+\alpha_{n}\right) x_{n+1}+\alpha_{n}(I-T-k I) x_{n+1}-(1-k) \alpha_{n} x_{n}+(2-k) \alpha_{n}^{2}\left(x_{n}-T x_{n}\right) \\
\quad & \quad+\alpha_{n}\left(T x_{n+1}-T x_{n}\right) .
\end{aligned}
$$

By $T q=q$, we have

$$
\begin{gathered}
x_{n}-q=\left(1+\alpha_{n}\right)\left(x_{n+1}-q\right)+\alpha_{n}(I-T-k I)\left(x_{n+1}-q\right)-(1-k) \alpha_{n}\left(x_{n}-q\right) \\
+(2-k) \alpha_{n}^{2}\left(x_{n}-T x_{n}\right)+\alpha_{n}\left(T x_{n+1}-T x_{n}\right) .
\end{gathered}
$$

By using the inequality (2), we obtain

$$
\begin{gathered}
\left\|x_{n}-q\right\| \geq\left(1+\alpha_{n}\right)\left\|x_{n+1}-q\right\|-(1-k) \alpha_{n}\left\|x_{n}-q\right\|-(2-k) \alpha_{n}^{2}\left\|x_{n}-T x_{n}\right\| \\
-\alpha_{n}\left\|T x_{n+1}-T x_{n}\right\| .
\end{gathered}
$$

Since

$$
\left\|T x_{n+1}-T x_{n}\right\| \leq L\left\|x_{n+1}-x_{n}\right\| \leq L(L+1) \alpha_{n}\left\|x_{n}-q\right\|,
$$

then

$$
\begin{gathered}
\left\|x_{n}-q\right\| \geq\left(1+\alpha_{n}\right)\left\|x_{n+1}-q\right\|-(1-k) \alpha_{n}\left\|x_{n}-q\right\|-(2-k) \alpha_{n}^{2}\left\|x_{n}-T x_{n}\right\| \\
-L(L+1) \alpha_{n}^{2}\left\|x_{n}-q\right\| .
\end{gathered}
$$


So,

$$
\begin{aligned}
\left\|x_{n+1}-q\right\| \leq[1+ & \left.(1-k) \alpha_{n}\right]\left(1+\alpha_{n}\right)^{-1}\left\|x_{n}-q\right\| \\
& +(2-k) \alpha_{n}^{2}\left(1+\alpha_{n}\right)^{-1}\left\|x_{n}-T x_{n}\right\| \\
& +L(L+1) \alpha_{n}^{2}\left(1+\alpha_{n}\right)^{-1}\left\|x_{n}-q\right\| \\
\leq[1+ & \left.(1-k) \alpha_{n}\right]\left(1-\alpha_{n}+\alpha_{n}^{2}\right)\left\|x_{n}-q\right\|+(2-k) \alpha_{n}^{2}\left\|x_{n}-T x_{n}\right\| \\
& \quad+L(L+1) \alpha_{n}^{2}\left\|x_{n}-q\right\| \\
\leq(1- & \left.k \alpha_{n}\right)\left\|x_{n}-q\right\|+M \alpha_{n}^{2}
\end{aligned}
$$

for some constant $M>0$, since $K$ is bounded. It follows from the Lemma of Dunn [7, p. 41] that the sequence $\left\{x_{n}\right\}$ strongly converges to the unique fixed point $q$. This completes the proof.

Remark. The nonlipschitzian version of Theorem 1 was proved in $[1,3]$ under the assumption that $X$ is a uniformly smooth Banach space by using the inequality

$$
\|x+y\|^{2} \leq\|x\|^{2}+2 \operatorname{Re}\langle y, J x\rangle+\max \{\|x\|, 1\}\|y\| \beta(\|y\|) .
$$

Theorem 2. Let $K$ and $T$ be as in Theorem 1. If $\alpha_{n}=\frac{k}{2\left(3+3 L+L^{2}\right)}$, where $k=$ $(t-1) / t,\{q\}=F(T)$, then the sequence $\left\{x_{n}\right\}$ generated by (3) converges strongly to the unique fixed point of $T$, and we have the estimate

$$
\left\|x_{n+1}-q\right\|<\rho^{n}\left\|x_{1}-q\right\|,
$$

where $\rho=1-\frac{k^{2}}{4\left(3+3 L+L^{2}\right)}$.

Proof. Since $0<\alpha_{n}<1$ and $T q=q$,

$$
\begin{aligned}
{\left[1+(1-k) \alpha_{n}\right]\left(1-\alpha_{n}+\alpha_{n}^{2}\right) } & =1-k \alpha_{n}+\alpha_{n}^{2}-(1-k) \alpha_{n}^{2}\left(1-\alpha_{n}\right) \\
& \leq 1-k \alpha_{n}+\alpha_{n}^{2}, \\
\left\|x_{n}-T x_{n}\right\| & \leq(1+L)\left\|x_{n}-q\right\| .
\end{aligned}
$$

By (4), we obtain

$$
\begin{aligned}
\left\|x_{n+1}-q\right\| & \leq\left(1-k \alpha_{n}\right)\left\|x_{n}-q\right\|+[1+(2-k)(1+L)+L(L+1)] \alpha_{n}^{2}\left\|x_{n}-q\right\| \\
& <\left[1-k \alpha_{n}+\left(3+3 L+L^{2}\right) \alpha_{n}^{2}\right]\left\|x_{n}-q\right\| \\
& =\left[1-\frac{k^{2}}{2\left(3+3 L+L^{2}\right)}+\frac{k^{2}}{4\left(3+3 L+L^{2}\right)}\right]\left\|x_{n}-q\right\| \\
& =\left[1-\frac{k^{2}}{4\left(3+3 L+L^{2}\right)}\right]\left\|x_{n}-q\right\| \\
& =\rho\left\|x_{n}-q\right\| .
\end{aligned}
$$

Hence $\left\|x_{n+1}-q\right\|<\rho^{n}\left\|x_{1}-q\right\|$. The proof is complete.

\section{REFERENCES}

1. X. Weng, Fixed point iteration for local strictly pseudocontractive mapping, Proc. Amer. Math. Soc. 113 (1991), 727-731. MR 92b:47099

2. C. E. Chidume, Iterative approximation of fixed points of Lipschitzian strictly pseudocontractive mappings, Proc. Amer. Math. Soc. 99 (1987), 283-288. MR 87m:47122

3. - Approximation of fixed points of strongly pseudocontractive mappings, Proc. Amer. Math. Soc. 120 (1994), 545-550. MR 94d:47056 
4. _ An iterative process for nonlinear Lipschitzian strongly accretive mappings in $L_{p}$ spaces, J. Math. Anal. Appl. 151 (1990), 453-461. MR 91k:47131

5. K-K. Tan and H-K. Xu, Iterative solutions to nonlinear equation of strongly accretive operators in Banach spaces, J. Math. Anal. Appl. 178 (1993), 9-21. MR 94g:47085

6. J. Bogin, On strict pseudo-contractions and a fixed point theorem, Technion preprint series No. MT-219, Haifa, Israel, 1974.

7. J. C. Dunn, Iterative construction of fixed points for multi-valued operators of the monotone type, J. Funct. Anal. 27 (1978), 30-50. MR 81f:47056

Department of Mathematics, Nanchang University, Nanchang, Jiangxi 330047, PeoPLE's Republic of China 